\title{
AN EXTENSION OF MacCORMACK'S METHOD FOR FLOWS WITH HIGHER-ORDER EQUATIONS AND IN DIFFERENT CONFIGURATIONS
}

\author{
S. J. YING and V. C. LIU \\ The University of Michigan, Ann Arbor, MI 48104, U.S.A. \\ (Received 9 September 1977; in revised form 4 April 1978)
}

\begin{abstract}
Aldract-The numerical scbeme for the computation of a shock discontinuity developed by MacCormack bas been extended to solve a number of differential equations, including cases explicitly containing higher-order derivatives: (1) Kortewes-de Vries equation with a term of third-order derivative, (2) a system of nonlinear equations governing nonsteady one-dimensional plasma fow in cylindrical coordinate, (3) equations of solar wind. Comparisons with previous results are made, if available, to illustrate the advantages of the present method. The question of convergence of the numerical calculation is discussed.
\end{abstract}

\section{INTRODUCTION}

The fundamental difficulties in treating time-dependent compressible flows stem from the large number of dependent variables, both hydrodynamic and thermodynamic, and the possible presence of shock discontinuities in the flow field. The difficult numerical study of shock waves, which are unique to the supersonic flows, has been directed towards smearing out the shock discontinuities while retaining accuracy away from the surface of discontinuity. Among the contemporary numerical techniques, Lax method[1] has the asset of simplicity hence is easier to be adopted in treating complex flow systems. Following Lax's initial study, MacCormack et al.[2-4], had made some improvements, especially in establishing a convergence criterion for the nonsteady one-dimensional flow in rectangular coordinates. MacCormack's method has generally been applied to solving a set of differential equations explicitly expressed only with first-order derivatives.

In the present study, we adopt the Lax-MacCormack technique to solving transient flow problems of more diversified configurations and physical situations. Consequently, the differential equations may involve, explicitly, second- and third-order derivatives, e.g. the propagation of a finite-amplitude water wave as described by the Korteweg-de Vries equation and the self-consistent (particle-field) study of plasma expansion where an electrostatic discontinuity is present. In the Korteweg-de Vries equation, the order of differentiation with respect to time is still of the first order but it is of the third order with respect to space coordinate. The result of our computation agrees well with previous work by Vliengenthart[S]. In the study of a plasma expansion, where flows of both ion and electron species are involved, the Poisson equation of the electric field must be taken into account. Thus the present numerical study takes on a new wrinkle. Expansion flows of cylindrical configurations is treated. Although Novak[6] has applied the MacCormack method to a flow problem in cylindrical coordinate, the differential equations treated are, however, of the first order. The convergency problem pertaining to the computations of plasma flows turns to be rather critical particularly with the cylindrical case. Finally we apply the method to the nonsteady spherical expansion flows of solar wind.

To ascertain the accuracy of the present method for equation in spherical coordinate we first solved two sets of linearized equations of second order and with different boundary conditions and compared the results of numerical computation with the analytical solutions. They are in very cood agreement. Then we applied the method to solve a solar wind problem which has been studied by Hundhausen and Gentry [7]. Hundhausen and Gentry used the particle-in-cell (PIC) method together with the combined Eulerian-Lagrangian scheme for solving the flare generated disturbance in the solar wind. As pointed out by Gentry et al. [8], the PIC method is accurate to the second order of the increment in space coordinate and to the first order of the 
time increment. The use of MacCormack method makes it possible to improve the accuracy of the functional time dependency. Since physical quantities may have rapid changes in many fluid problems, the MacCormack method which is accurate to the second order of time increment can save some computation time for reaching the same accuracy.

\section{NUMERICAL TECHNIQUE}

Let the differential equations in question be written in the following form

$$
f_{t}=a f_{r}+b f_{n}+c f_{m}+s
$$

where $f$ and $s$ are column vectors representing the dependent variables and the "source-like" terms, respectively, in the governing equations. Following MacCormack's scheme, from given initial values of $f_{j}^{n}$ at the time $t$, the predicted values, denoted by $f_{j}^{\overline{n+1}}$, are computed from

$$
\begin{aligned}
\frac{f_{i}^{\overline{n+1}}-f_{i}^{n}}{\Delta_{n} t}= & a_{j}^{n} \frac{f_{i+1}^{n}-f_{i}^{n}}{\Delta r}+b_{j}^{n} \frac{1}{(\Delta r)^{2}}\left(f_{j+1}^{n}+f_{j-1}^{n}-2 f_{j}^{n}\right) \\
& +c_{j}^{n} \frac{1}{(\Delta r)^{3}}\left(f_{j+2}^{n}+3 f_{j}^{n}-3 f_{j+1}^{n}-f_{j-1}^{n}\right)+s_{j}^{n}
\end{aligned}
$$

where the subscript denotes a physical position, and the superscript denotes the time. Rearranging the equation, we ortain the predicted values

$$
\begin{aligned}
f_{j}^{\overline{n+1}}= & f_{j}^{n}+\lambda a_{j}^{n}\left(f_{j+1}^{n}-f_{i}^{n}\right)+\lambda b_{j}^{n} \frac{1}{\Delta r}\left(f_{j+1}^{n}+f_{i-1}^{n}-2 f_{j}^{n}\right) \\
& +\lambda c_{j}^{n} \frac{1}{(\Delta r)^{2}}\left(f_{j+2}^{n}+3 f_{j}^{n}-3 f_{j+1}^{n}-f_{j-1}^{n}\right)+\Delta_{n} t s_{j}^{n}
\end{aligned}
$$

where

$$
\lambda=\frac{\Delta_{n} t}{\Delta r}
$$

The final corrected values at $t+\Delta_{n} t, f_{j}^{n+1}$, is the average of $f_{j}^{n}$ and the predicted values at

$$
t=\sum_{n=1}^{n+2} \Delta_{n} t \text { denoted by } \overline{f_{i}^{\overline{n+2}}}
$$

$f_{i}^{\overline{n+2}}$ is obtainable from eqn (2) with backward difference in $r$ for first and third derivatives. Hence

$$
f_{j}^{n+1}=\frac{1}{2}\left(f_{i}^{n}+f_{i}^{\overline{n+2}}\right)
$$

where

$$
\begin{aligned}
f_{i}^{\overline{n+2}}= & f_{i}^{\overline{n+1}}+\lambda a_{i}^{\overline{n+1}}\left(f_{i}^{\overline{n+1}}-f_{i-1}^{\overline{n+1}}\right)+\frac{\lambda}{\Delta r} b_{i}^{\overline{n+1}}\left(\overline{f_{i+1}^{n+1}}+f_{i-1}^{\overline{n+1}}-2 f_{i}^{\overline{n+1}}\right) \\
& +\frac{\lambda}{(\Delta r)^{2}} c_{i}^{\overline{n+1}}\left(\overline{f_{i+1}^{n+1}}+3 \overline{f_{i-1}^{\overline{n+2}}}-3 f_{i}^{\overline{n+1}}-\overline{f_{i-2}^{n+1}}\right)+\Delta_{n} t s_{i}^{\overline{n+1}} .
\end{aligned}
$$

\section{KORTEWEG-DE VRIES EQUATION}

To apply the MacCormack scheme to differentia equations with higher order, the Korteweg-de Vries equation is used as a test case. The $\mathrm{KdV}$ equation for water waves can be written as [5]

$$
\frac{\partial \eta}{\partial t}+\alpha \eta \frac{\partial \eta}{\partial x}+\beta \frac{\partial^{3} \eta}{\partial x^{3}}=\mu \frac{\partial^{2} \eta}{\partial x^{2}}
$$


where $\eta$ is the dimensionless magnitude of variation; $\alpha, \beta, \mu$ are dimensionless coefficients; $x, t$ are dimensionless space and time coordinate. Initial conditions used in the computation are

$$
\begin{array}{ll}
\eta=1 & \text { as } x<0 \\
\eta=0 & \text { as } x>0 .
\end{array}
$$

Boundary condition at large $x$ is assumed as

$$
\left(\frac{\partial \eta}{\partial x}\right)_{n}=\frac{1}{2}\left[\left(\frac{\partial \eta}{\partial x}\right)_{n-1}+\left(\frac{\partial \eta}{\partial x}\right)_{n+1}\right]
$$

Quantities used in the computation are $\Delta x=0.2, \Delta t=0.015, \alpha=0.1, \beta=0.1, \mu=0,0.01$ and 0.1. The results are shown in Figs. 1-4. The curves shown in Figs. 1 and 2 for the case of $\alpha=0.1, \beta=0.1$ and $\mu=0$ at $t=1.5$ and $t=6.0$ agree exactly with Fig. 11 in the work of Vliegenthart[5].

\section{PLASMA EXPANSION INTO AN AXIALLY SYMMETRIC VACUUM SPACE}

To extend the MacCormack scheme to differential equations involving Poison's equation, the expansion of uniform plasma into a vacuum is studied. Suppose that the plasma is initially contained in the axially symmetric space $r>a$. At $t=0$, the plasma expands into the vacuum space $0<r<a$. The governing equations are written as follows

$$
\frac{\mathrm{d} u}{\mathrm{~d} t}=-\frac{\partial \phi}{\partial r}
$$

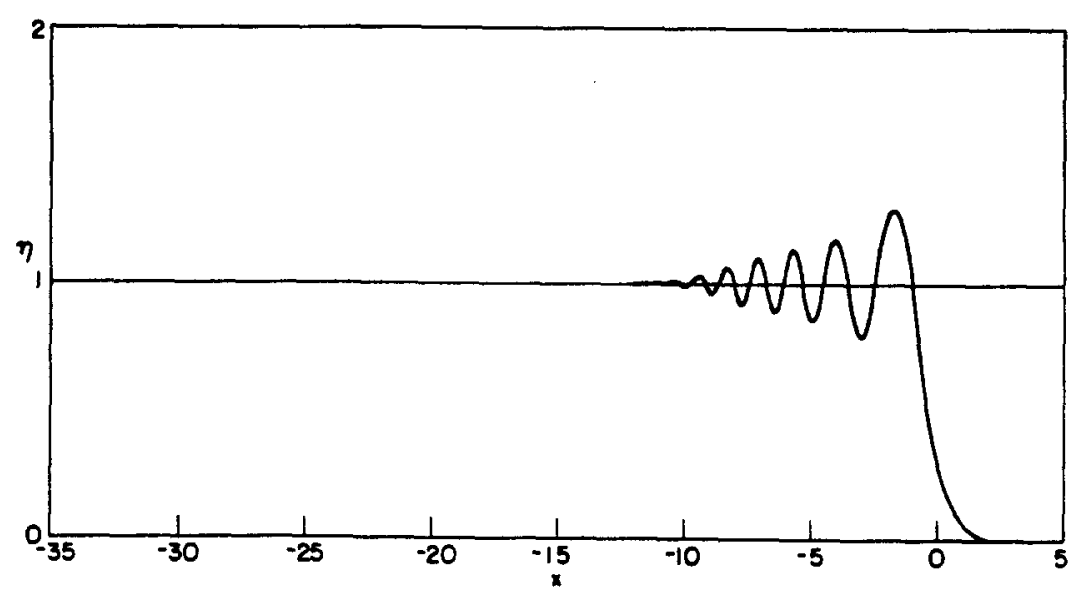

Fig. 1. Solution of $\mathrm{KdV}$ equation with $\alpha=0.1, \beta=0.1, \mu=0$ at $t=1.5$.

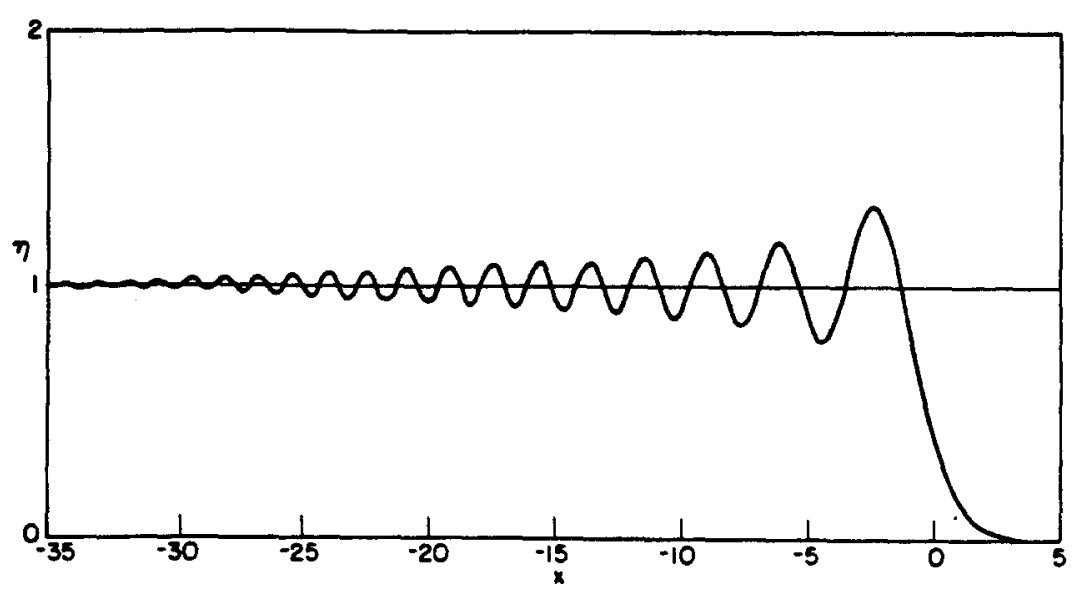

Fig. 2. Solution of $\mathrm{KdV}$ equation with $a=0.1, \beta=0.1, \mu=0$ at $t=6.0$. 


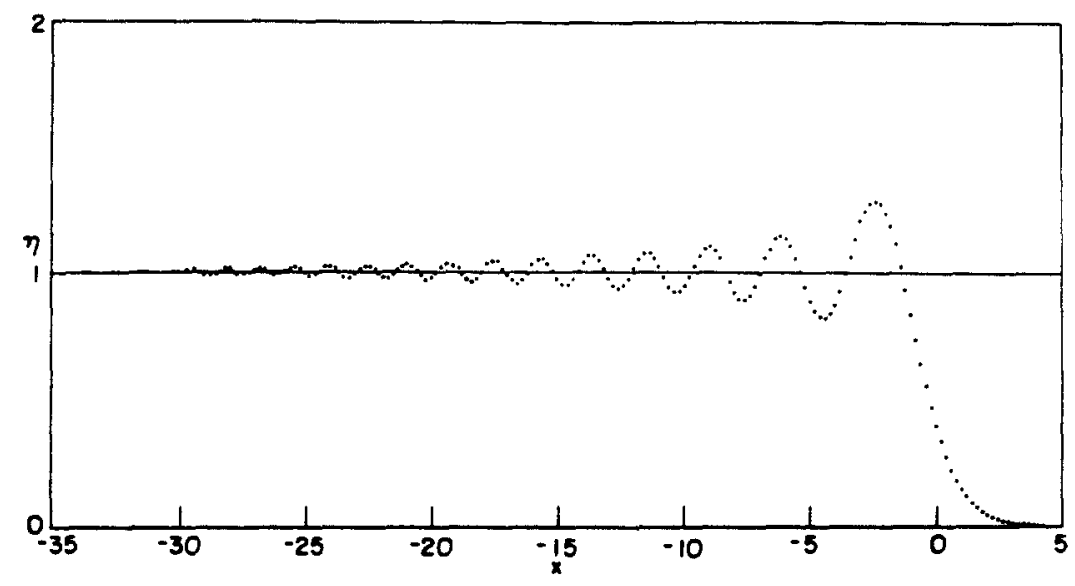

Fig. 3. Solution of $\mathrm{KdV}$ equation with $\alpha=0.1, \beta=0.1, \mu=0.01$ at $t=6.0$.

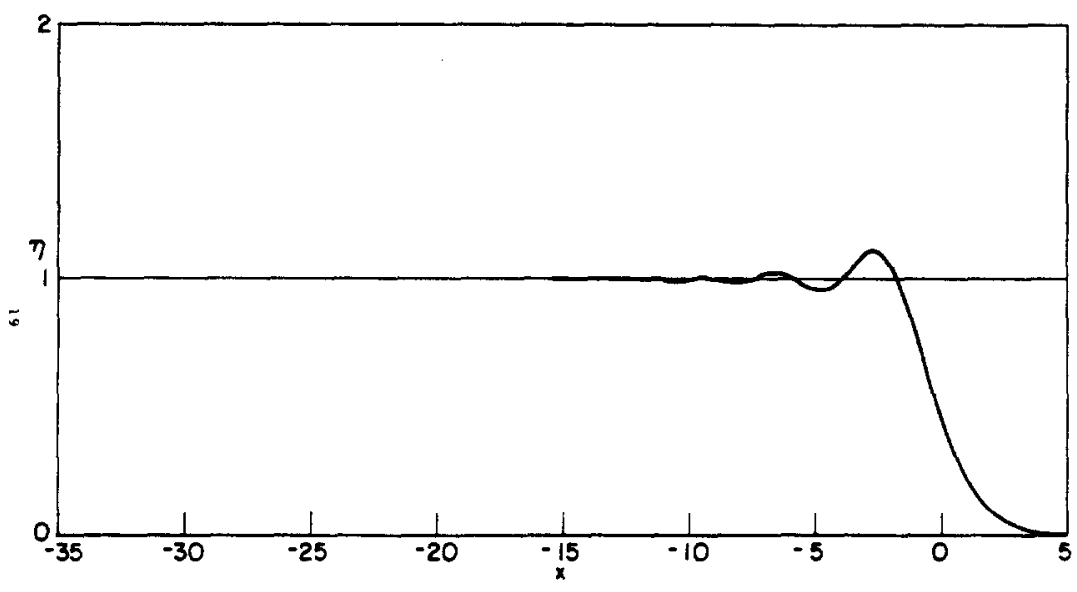

Fig. 4. Solution of KdV equation with $\alpha=0.1, \beta=0.1, \mu=0.1$ at $t=6.0$.

$$
\begin{gathered}
\frac{\mathrm{d} r}{\mathrm{~d} t}=u \\
\frac{1}{N} \frac{\mathrm{d} N}{\mathrm{~d} t}=-\frac{\partial u}{\partial r}-\frac{u}{r} \\
\frac{\partial^{2} \phi}{\partial r^{2}}+\frac{1}{r} \frac{\partial \phi}{\partial r}=\mathrm{e}^{\phi}-N
\end{gathered}
$$

where $u=$ the dimensionless velocity; $\phi=$ the dimensionless potential; $N=$ the dimensionless particle density of ion; $r=$ the dimensionless radius; $t=$ the dimensionless time. Initial conditions used are

$$
\begin{array}{ll}
N=1 & \text { as } r>r_{f}, \\
N=0 & \text { as } r<r_{f}, \\
u=0 & \text { for all } r .
\end{array}
$$

And boundary conditions at the wave front, $r=r_{f}$,

$$
\begin{gathered}
\phi=\ln \frac{8 A^{2}}{\left(1-A^{2} r_{f}^{2}\right)^{2}} \\
\frac{\partial \phi}{\partial r}=\frac{4 A^{2} r_{f}}{1-A^{2} r_{f}^{2}}
\end{gathered}
$$


where $A^{2}=\exp \left(\phi_{0}\right) / 8$ and $\phi_{0}$ is the potential at $r=0$. Boundary conditions of $\phi$ and $\partial \phi / \partial r$ at $r \rightarrow \infty$ are expected to be zero.

To solve the above set of equations, the value of $A$ is adjusted by satisfying the condition that the $\phi$ and $\partial \phi / \partial r$ approach zero at large $r$. The value of $\phi$ in the region $r>r_{f}$ is determined by the Taylor's series expansion and in the region $r<r_{f}$ is obtained by the analytical solution

$$
\phi=\ln \frac{8 A^{2}}{\left(1-A^{2} r^{2}\right)^{2}} .
$$

With the potential $\phi$ obtained, the values of $u$ and $r$ are determined with the use of the MacCormack's scheme. Finally the particle density $N$ is integrated. Thus, by repeating the above procedures the variables are obtained at successive time steps.

The convergence criterion is found such that as the time increment is determined by

$$
\Delta t=C_{\text {sf }} \frac{\Delta r}{\|u\|_{\max }}
$$

$C_{s f}$ must be less than or equal to 1 . The results shown in Figs. 5-8 are computed with $\Delta r=0.025$ and $C_{s f}=1$.

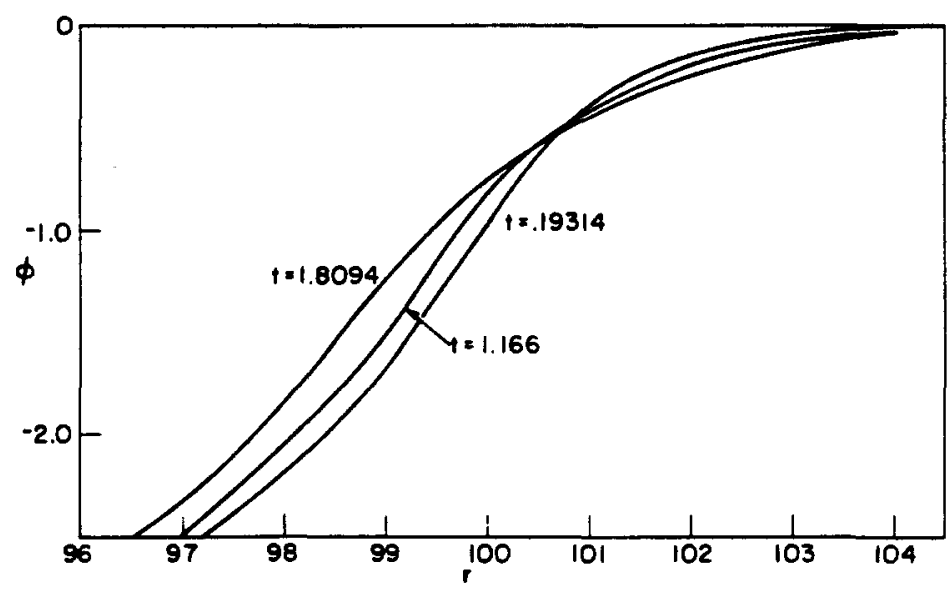

Fig. 5. Distributions of dimensionless potential.

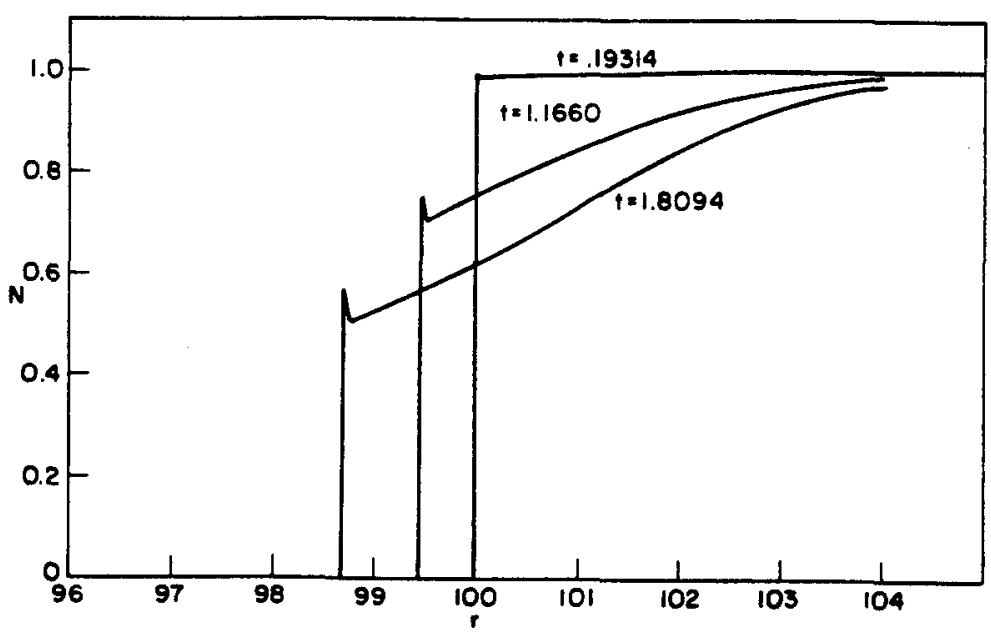

Fig. 6. Distributions of dimensionless particle density.

\section{VERIFICATION OF THE MACCORMACK METHOD}

To make it certain that the MacCormack's method is applicable to non-steady spherically symmetrical flow problems, two cases with linearized equations are studied first. The physical meanings and the corresponding equations are given as follows. 


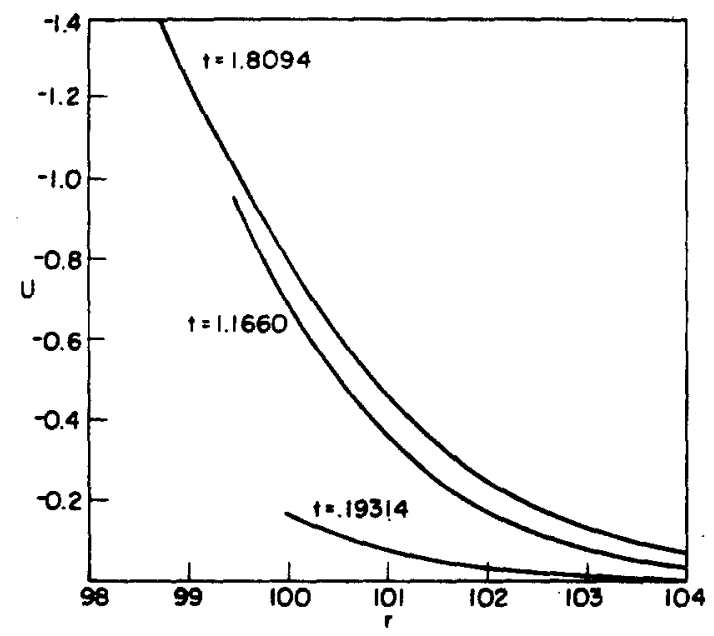

Fig. 7. Distributions of dimensionless velocity.

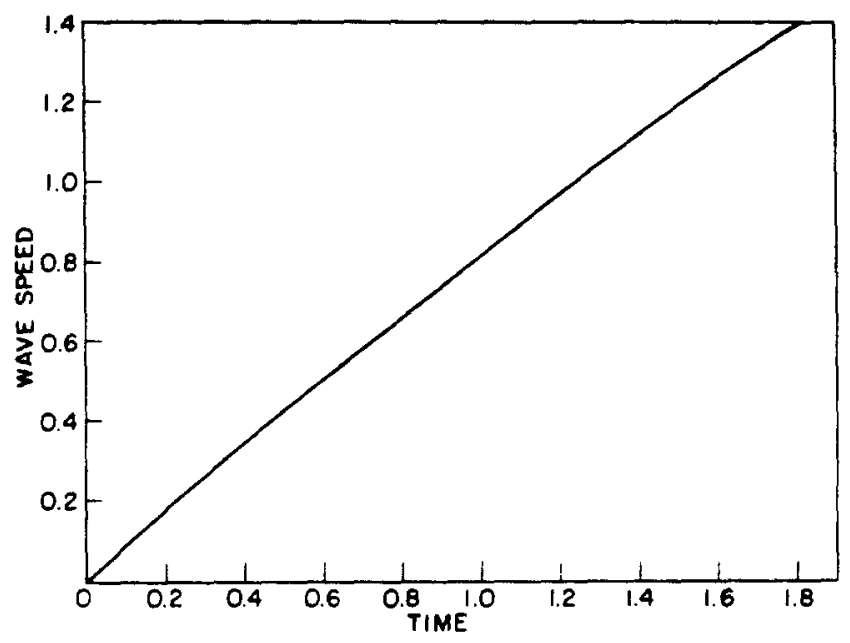

Fig. 8. Change of wave speed.

(a) Linearized equations without source

Consider a sound wave propagating spherically into undisturbed medium. Equations can be written as

$$
\begin{gathered}
\frac{\partial p}{\partial t}+\rho_{0}\left(\frac{\partial u}{\partial r}+\frac{2 u}{r}\right)=0 \\
\frac{\partial u}{\partial t}=-\frac{a^{2}}{\rho_{0}} \frac{\partial p}{\partial r}
\end{gathered}
$$

where $\rho_{0}, \rho$ are densities of undisturbed and disturbed medium respectively, $u$ the velocity, $a$ the speed of sound, $t$ the time and $r$ the radius.

(b) Linearized equations with sources

Consider that a sound wave propagates spherically into a medium under chemical reaction. The equations then can be written as

$$
\begin{gathered}
\frac{\partial p}{\partial t}+\rho_{0}\left(\frac{\partial u}{\partial r}+\frac{2 u}{r}\right)=\dot{\omega} H\left(\frac{r_{0}+a t-r}{a}\right) \\
\frac{\partial u}{\partial t}+\frac{a^{2}}{\rho_{0}} \frac{\partial p}{\partial r}=\dot{m} H\left(\frac{r_{0}+a t-r}{a}\right)
\end{gathered}
$$


where $\dot{\omega}$ is the mass source, in the momentum sink and $H\left[\left(r_{0}+a t-r\right) / a\right]$ is a step function such that

$$
H\left(\frac{r_{0}+a t-r}{a}\right)= \begin{cases}1 & \text { as } r<\left(r_{0}+a t\right) \\ 0 & \text { as } r>\left(r_{0}+a t\right)\end{cases}
$$

in which $r=r_{0}$ is the surface where the sound wave starts.

The results of numerical computation are described separately for the two cases.

(a) To illustrate the numerical computation works for the cases of discontinuity across the wave front, the initial and boundary conditions are so chosen such that $\rho=\rho_{0}, u=0$ in the region $r_{0}<r<\infty$ at $t=0$ and

$$
\begin{gathered}
\rho=\rho_{0}+A H(t) \text { at } r=r_{0} \\
u=\frac{a A}{\rho_{0} r_{0}}\left(r_{0}+a t\right) H(t) \text { at } r=r_{0}
\end{gathered}
$$

where $A$ is a constant, $|A|<\rho_{0}$ and $H(t)=1$ as $t>0$ and $H(t)=0$ as $t<0$. It is necessary that the velocity $u$ be expressed as above for satisfying the differential eqns $(17 \mathrm{a}, \mathrm{b})$ and $(18 \mathrm{a}, \mathrm{b})$. The analytical solution of eqns $(17 a, b)$ is

$$
\begin{gathered}
\rho=\rho_{0}+A \frac{r_{0}}{r} H\left(\frac{r_{0}+a t-r}{a}\right) \\
u=\frac{a A r_{0}}{\rho_{0} r^{2}}\left(r_{0}+a t\right) H\left(\frac{r_{0}+a t-r}{a}\right) .
\end{gathered}
$$

The results of numerical computation and the analytical solution are shown in Figs. 9 and 10. The numerical values of physical quantities used in the calculation are given as follows

$$
\begin{gathered}
\rho_{0}=0.00125 \mathrm{fm} / \mathrm{cm}^{3}, \quad A=0.001 \rho_{0}, \\
a=3.40180 \times 10^{4} \mathrm{~cm} / \mathrm{sec}, \quad r_{0}=100 \mathrm{~cm}, \\
\Delta t=0.9 \frac{\Delta r}{a}, \Delta r=1 \mathrm{~cm}, \\
t=0.0016932 \mathrm{sec} .
\end{gathered}
$$

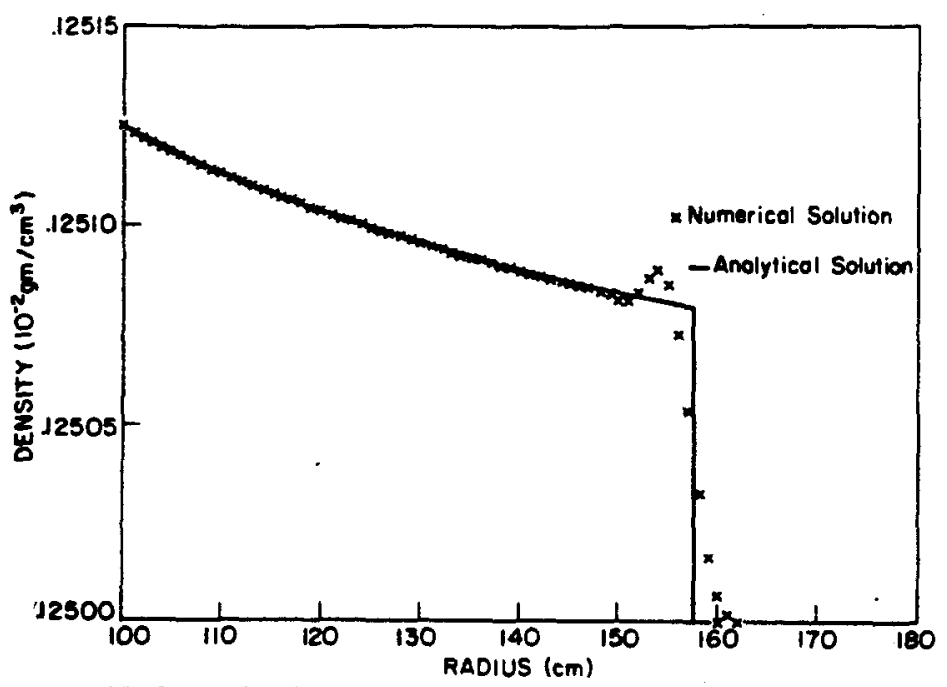

Fig. 9. Density distribution of linearized equations without source. 


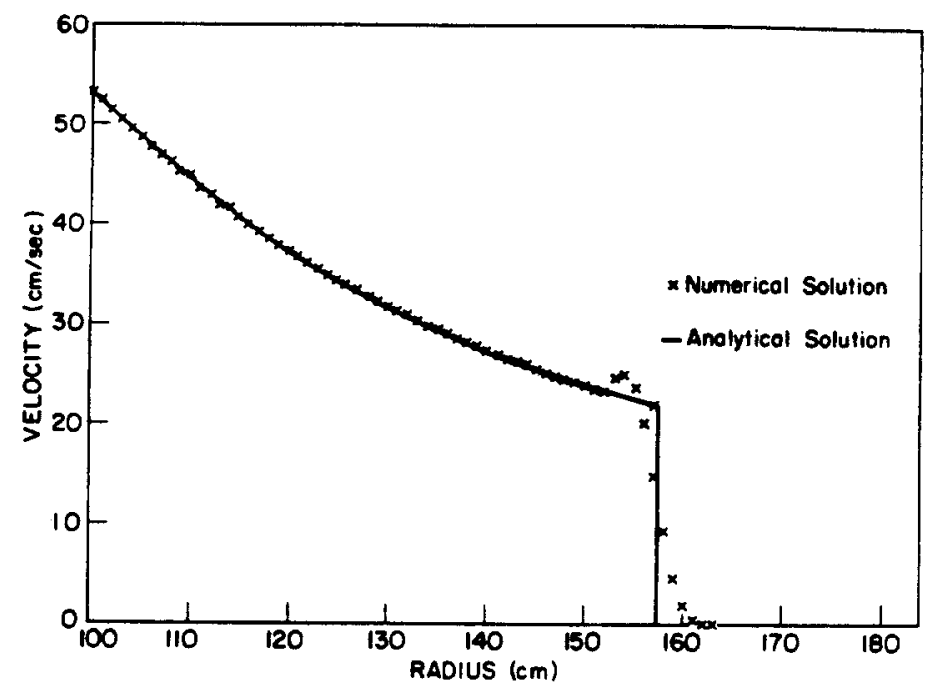

Fig. 10. Velocity distribution of linearized equations without source.

(b) The analytical solution of eqns $(14 a, b)$ are

$$
\begin{aligned}
& \rho=\rho_{0}+A \frac{r_{0}}{r} H\left(\frac{r_{0}+a t-r}{a}\right)+\frac{\rho_{0} \beta}{a}\left[\left(r-r_{1}\right) H\left(r-r_{1}\right)-\left(r-r_{2}\right) H\left(r-r_{2}\right)\right] H\left(\frac{r_{0}+a t-r}{a}\right) \\
& u=\frac{a A r_{0}}{\rho_{0} r^{2}}\left(r_{0}+a t\right) H\left(\frac{r_{0}+a t-r}{a}\right)+\frac{\beta}{a}\left[\left(r-r_{1}\right) H\left(r-r_{1}\right)-\left(r-r_{2}\right) H\left(r-r_{2}\right)\right] H\left(\frac{r_{0}+a t-r}{a}\right)
\end{aligned}
$$

as

$$
\begin{gathered}
\dot{m}=\beta\left[H\left(r-r_{1}\right)-H\left(r-r_{2}\right)\right] \\
\dot{\omega}=\frac{\rho_{0} \beta}{a}\left[\left(3-\frac{2 r_{1}}{r}\right) H\left(r-r_{1}\right)-\left(3-\frac{2 r_{2}}{r}\right) H\left(r-r_{2}\right)\right] .
\end{gathered}
$$

In the numerical computation, the additional quantities used are

$$
\begin{gathered}
\beta=10^{-4} a^{2}=11.57224 \times 10^{4} \mathrm{~cm} / \mathrm{sec}^{2} \\
r_{1}=139 \mathrm{~cm}, \quad r_{2}=144 \mathrm{~cm} . \\
t=0.0018306 \mathrm{sec} . \quad \Delta t=0.99 \frac{\Delta r}{a} .
\end{gathered}
$$

The comparison between the numerical and the analytical solutions is given in Figs. 11 and 12 .

From Figs. 9-12, we can easily see that the numerical solutions agree very well with the analytical solution. That means the MacCormack method does work for the spherical coordinate. The numerical calculations were done using the time step of

$$
\Delta T=C_{S F} \frac{\Delta r}{a}
$$

It is found that the results converge to the analytical solution as $C_{S F}<1$.

\section{SOLAR WIND}

While disturbances are generated by flares on the solar surface, spherical shock waves are often propagating radially into existing solar wind. This physical condition is ideal for applying the MacCormack method. 


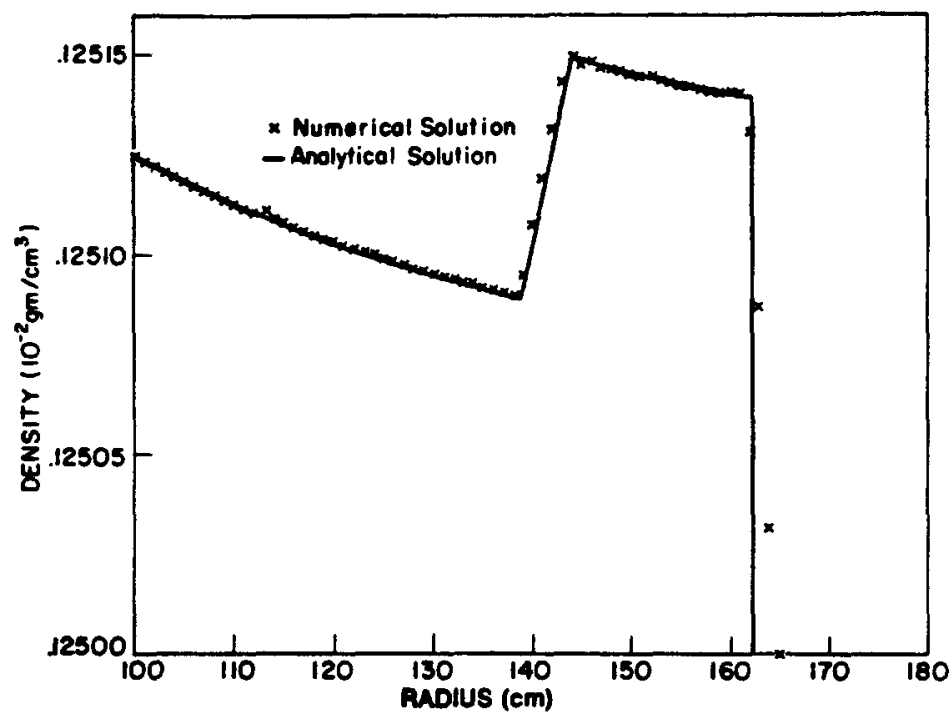

Fig. 11. Density distribution of linearized equations with sources.

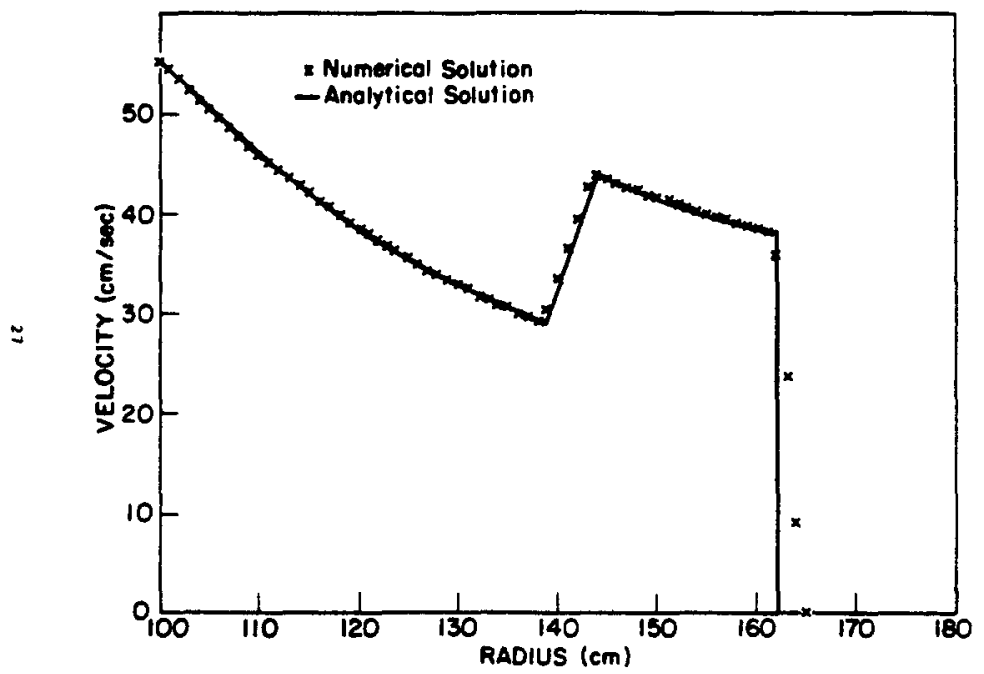

Fig. 12. Velocity distribution of linearized equations with sources.

Consider an unsteady disturbance propagating outward radially into an inviscid, adiabatic medium which is a perfect gas, the gravitational force is opposite to the radial direction, and mass source, momentum sink and energy source are allowed in the medium. The derivation of equations is omitted here since it is not of our primary interest. The basic equations without source terms are given in the paper by Hundhausen and Gentry [7]. The governing equations in dimensionless form can be written as follows:

$$
\begin{gathered}
\frac{\partial n}{\partial t}=-\alpha u \frac{\partial n}{\partial R}-n\left(\alpha \frac{\partial u}{\partial R}+\frac{2 u}{r}\right)+\dot{n} \\
\frac{\partial u}{\partial t}=-\alpha u \frac{\partial u}{\partial R}-\alpha \frac{\partial T}{\partial R}-\alpha \frac{T}{n} \frac{\partial n}{\partial R}-\frac{G}{r^{2}}-\dot{m} \\
\frac{\partial T}{\partial t}=-\alpha u \frac{\partial T}{\partial R}-\frac{2}{3} T\left(\alpha \frac{\partial u}{\partial R}+\frac{2 u}{r}\right)+\frac{2}{3} \dot{q}
\end{gathered}
$$

where $n=$ the dimensionless particle density; $u=$ the dimensionless radial velocity: $T=$ the dimensionless temperature; $r=$ the dimensionless radius: $t=$ the dimensionless time: $G=$ the 
dimensionless gravitational constant; $\dot{n}, \dot{m}, \dot{q}=$ the dimensionless mass source, momentum sink and energy source, respectively.

For the present purpose where the variations of $n(r, t), u(r, t)$ and $T(r, t)$ are of interest only, the absolute values of them are of no concern. Since many physical quantities are expected to vary with the exponential function of the dimensionless radius $r$, it is desirable to introduce the new space variables

$$
R=\frac{1}{A} \ln \frac{r}{B}
$$

and

$$
\alpha=\frac{\mathrm{d} R}{\mathrm{~d} r}=\frac{1}{A B} \mathrm{e}^{-A R} .
$$

In the numerical computation, the steady state solution has been used to prescribe the initial values of $n, u$ and $T$. The values at the inner boundary are specified according to RankineHugoniot relations for possible shock discontinuity. At the outer boundary, values are calculated by assuming that the slope of $f_{j}$ is the average of slopes at $j-1$ and $j+1$, i.e.

$$
f_{i}^{n}-f_{i-1}^{n}=\frac{1}{2}\left[\left(f_{i+1}^{n}-f_{j}^{n}\right)+\left(f_{i-1}^{n}-f_{j-2}^{n}\right)\right]
$$

Hence

$$
f_{j+1}^{n}=f_{j-2}^{n}+3 f_{i}^{n}-3 f_{j-1}^{n} \text {. }
$$

This relation has been used for both the predicted step and the corrected step.

It is of interest to test for the convergence criterion of the present computation. Now that the time step

$$
\Delta t=C_{s f} \frac{\Delta R}{(u+a)_{\max }}
$$

we found that the results converge as $C_{s f}<1$.

The steady state solution of solar wind obtained by Whang[9] is used as our initial condition. For convenience equation forms approximately matched to Whang's result are used. However, they are checked through our numerical computations, the result of 30 time step computation shows that they represent the steady state solution. The equations of our initial conditions are

$$
\begin{aligned}
n & =r^{-3} \\
u & =0.107676(30)^{\left(1 .-r^{-0.4343)}\right.} \\
T & =r^{-0.4346} \\
\dot{q} & =1.77808 \times 10^{-2} r^{-0.6343} \\
\dot{m} & =\dot{n}=0 .
\end{aligned}
$$

Constants used in the calculations are

$$
\begin{aligned}
G & =4.82643 \\
A & =0.238198 \\
B & =0.788046 \\
C_{s f} & =0.8 \\
\Delta R & =0.5 .
\end{aligned}
$$


At the inner boundary the shock strength for the computation are

$$
\begin{gathered}
n=1+3 \sin t, \\
u=0.107676+0.430704 \sin t, \\
T=1+24 \sin t .
\end{gathered}
$$

The results of numerical computation without mass source and momentum sink are shown in Figs. 13-15. These curves are compared with the results of Hundhausen and Gentry, they are in

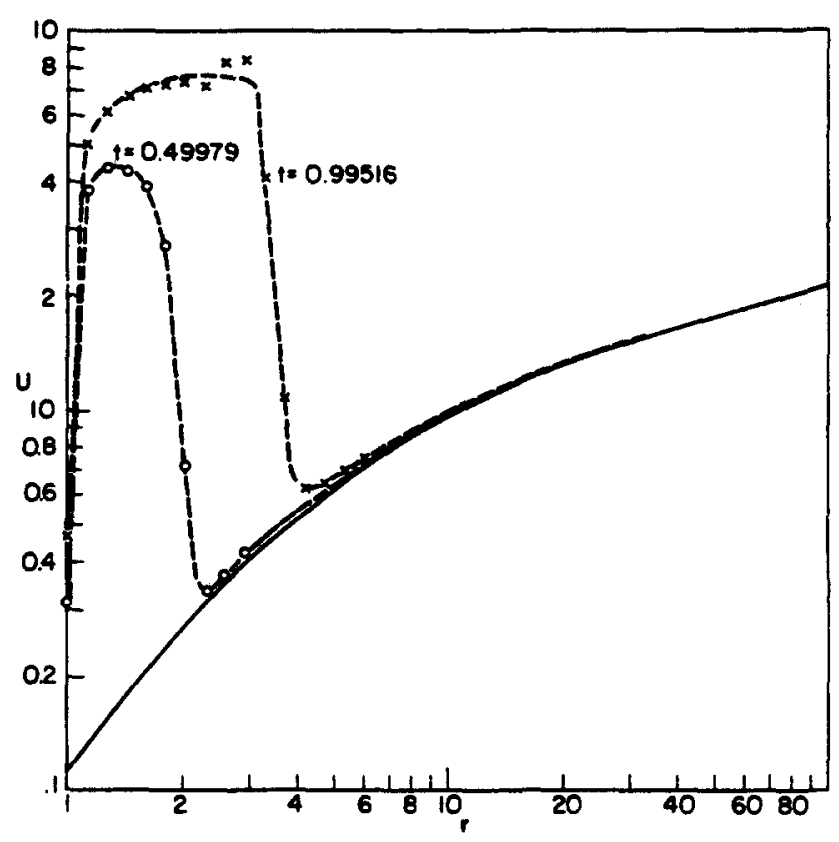

Fig. 13. Dimensionless velocity distributions.

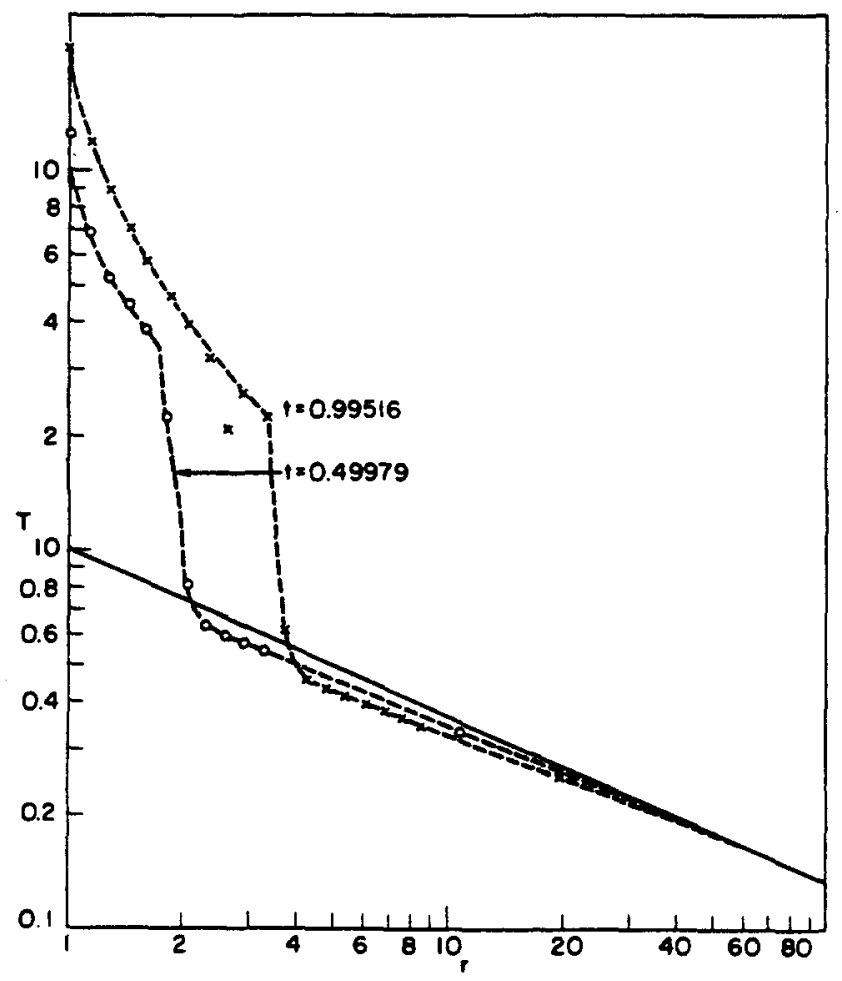

Fig. 14. Dimensionless temperature distributions. 


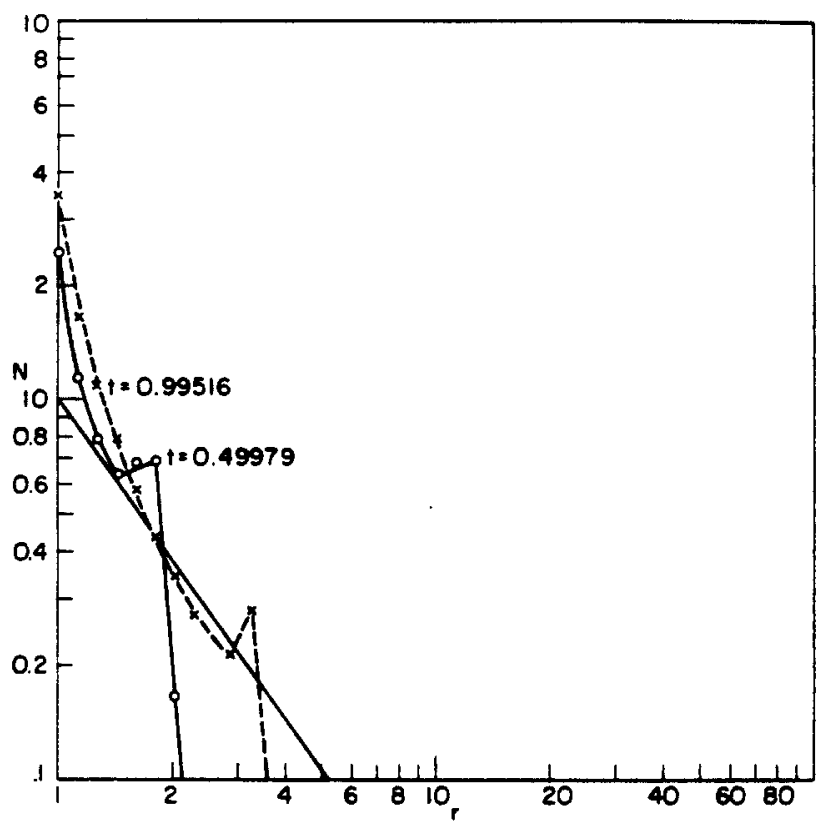

Fig. 15. Dimensionless particle density distributions.

good agreement qualitatively. No quantitative comparison could be made, because the physical quantities used in their calculations are not completely given in their paper.

\section{CONCLUSION}

From this study we can conclude that the MacCormack method can be applied to generalized 1-dimensional compressible fluid flow with rapid variation. The method is successful for equations with third order differentiation with respect to space coordinate as in the case of the $\mathrm{KdV}$ equation and the coupled equations of plasma flows.

The procedure of numerical computation appears simpler than many existing methods, e.g. PIC method, and the accuracy of each step in computation is to the second order of $\Delta t$.

The convergence criteria are that with $\Delta t$ calculated from eqns (16), (23) and (27), $C_{2 f}$ usually must be less than 1 . This point agrees with the previous works by MacCormack et al. [4] and Novak [6].

The present study has been supported by the NASA Research Grant NGR 23-005-094.

\section{REFERENCES}

1. P. D. Lax, Weak solutions of nonlinear hyperbolic equations and their numerical computation. Commun. Pure Appl. Math. 7, 159-193 (1954).

2. R. W. MacCormack and A. J. Paullay, Computational efficiency achieved by time splitting of finite difference operators. AIAA Paper 72-154 (1972).

3. R.W. MacCormack, The numerical solution of the interaction of a shock wave with a laminar boundary layer. Proc. 2nd Int. Conf. Numerical Methods in Fluid Dynamics, pp. 151-163. Springer-Verlag, Berlin (1971).

4. R. W. MacCormack and A. J. Paulley, The infuence of the computational mesh on accuracy for initial value problems with discontinuous or non-unique solutions. Computer and Fuids 2, 339-361 (1974).

5. A. C. Vliegenthart, On finite-difference methods for the Korteweg-de Vries Equation. J. Engng Math. 5, 137-155 (1971).

6. J. M. Novak, A numerical study of the effects of intense transient heating in a Laval nozzle. Ph.D. dissertation. University of Michigan (1974).

7. A. J. Hundhausen ind R. A. Gentry, Numerical simulation of flare-senerated disturbances in the solar wind. J.G.R. Space Phys. 74 (11), 2908-2918 (1969).

8. R. A. Gentry, R. E. Martin and B. J. Daly, An Eulerian differencing method for unsteady compressible flow problems. J. Comp. Phys. 1, 87-118 (1966).

9. Y. C. Whang, Conversion of magnetic field energy into kinetic energy in the solar wind. Proc. Conf. on Solar Wind. 236-243, NASA SP-308, Pacific Grove, California (1971). 